\title{
Conditional production of acyl-homoserine lactone-type quorum-sensing signals in clinical isolates of enterobacteria
}

Single-celled bacteria exchange small, self-produced chemical molecules to monitor their population density and control a variety of physiological functions in a cell-density-dependent manner, a process called quorum sensing (Fuqua et al., 1994; Waters \& Bassler, 2005). Many

Gram-negative bacteria use

acyl-homoserine lactones (AHLs) as cell-cell communication molecules. In these LuxR-LuxI-type quorum-sensing systems, the AHL signalling molecule is usually synthesized by a protein related to the LuxI protein of Vibrio fischeri and binds to an intracellular receptor protein that resembles the $V$. fischeri LuxR protein, which activates transcription of target genes (Whitehead et al., 2001). Several quorum-sensing systems are also extremely important to human health, as they regulate virulence determinants in bacterial pathogens. One example involves the LasR/ LasI, RhlR/RhlI quorum-sensing systems of the human pathogen Pseudomonas aeruginosa (Venturi, 2006). In the Enterobacteriaceae, however, only a few AHL-based quorum-sensing systems, such as in Yersinia (Atkinson et al., 2006) and Serratia (Daniels et al., 2004), have been reported, whilst many other members of the family, including Escherichia and Salmonella, are generally thought to produce and utilize non-AHL signal molecules (Kaper \& Sperandio, 2005; Xavier \& Bassler, 2003). All quorum-sensing systems in enterobacteria identified so far control different physiological functions related to their pathogenesis.

Many species in the Enterobacteriaceae can be found in various environments and are often commensal in human intestines.

However, these organisms can cause infections, with the hospital environment being one of the major settings. The factors that provoke commensal bacteria to become nosocomial pathogens are largely unknown and cell-cell communication has been speculated as being involved in this process (Kaper \& Sperandio, 2005). In this study, we examined AHL-like quorum signal production in 53 strains belonging to the Enterobacteriaceae, isolated from blood samples of patients with high fever admitted to the Sir Run Run Shaw Hospital in Hanzhou, China. We found that diverse AHL signals were produced in many of these clinical isolates under different growth conditions, indicating that AHL production exists in a wide variety of enterobacteria capable of causing infections. This highlights the importance of future studies of the relationship between quorum sensing and pathogenesis of enterobacterial species.

\section{Strains were grown using rich} Luria-Bertani (LB) medium or minimal medium (M9 supplemented with $0 \cdot 5 \%$ glucose) under aerobic (shaking) and microaerophilic conditions (stationary at $0.5 \mathrm{MPa}$ air pressure) at $37^{\circ} \mathrm{C}$ until cultures reached a high cell density $\left(\mathrm{OD}_{600}>2 \cdot 0\right)$. Cell-free supernatants were subjected to AHL bioassays (Zhu et al., 2003). Supernatants from 44 strains had no detectable AHL activity under any of the growth conditions, whilst supernatants from nine strains displayed various AHL activities under at least one of the growth conditions (Table 1). Among these, three strains (Enterobacter cloacae, Citrobacter freundii and Serratia marcescens) produced significant amounts of AHLs under all four conditions tested. Two Klebsiella pneumoniae strains, one Klebsiella oxytoca strain and one Enterobacter aerogenes strain produced high AHL activities only when grown microaerophilically in LB medium. One S. marcescens strain (SRRSH67) produced more AHLs in rich media, whilst strain SRRSH69 preferred aerobic growth conditions for AHL production. To visualize the AHL contents produced by the above strains, a TLC analysis (Shaw et al., 1997) was performed by applying ethyl acetate-extracted supernatants to C18 reversed-phase TLC plates (Fig. 1). Most of the strains produced more than one type of AHL, whilst retaining production of the same AHLs, albeit at different levels, under different growth conditions. This indicated that the expression of AHL synthases in these strains may have been affected by the growth conditions. One exception was seen in S. marcescens SRRSH68, where it produced an extra AHL when grown in LB aerobically, suggesting that multiple AHL synthases are involved.

To evaluate the role that quorum sensing plays in pathogenesis, it is necessary to obtain mutations in quorum-sensing components that disrupt normal quorum-sensing functions. Here, we developed a simple, high-throughput screening method to identify genes involved in AHL synthesis in enterobacteria. We introduced mariner transposon (Rubin et al., 1999) insertions into S. marcescens SRRSH67. The transposon mutants were grown in 96-well plates and then overlaid with agar media containing AHL bioassay strains and X-Gal. After incubation, X-Gal hydrolysis (visible as a blue halo) was examined. From 6000 mutants, we obtained one transposon insertion mutant that failed to produce AHLs in our 96-well assay (producing a white halo). This strain (SRRSH67-1) did not produce AHLs in the standard liquid AHL bioassay (Fig. 2a) and no AHL was detected in a TLC assay

(Fig. 2b). Arbitrary PCR (Judson \& Mekalanos, 2000) and sequence analysis were performed to determine the transposon insertion. The insertion was in an open reading frame encoding a protein homologous to the AHL synthase SwrI of Serratia liquefaciens (93\% identity) (Eberl et al., 1996). Interestingly, quorum sensing may negatively regulate motility in this clinical isolate, as the AHL-deficient mutant was more motile than the wild-type on $0 \cdot 4 \%$ agar plates (Fig. 2c). It has been reported that, in S. marcescens SS-1, quorum sensing also negatively regulates motility through SpnR/SpnI (Horng et al., 2002).

Taken together, this study examined 53 clinical isolates of enterobacteria and found 
Table 1. Clinical isolates of enterobacteria grown under different conditions produced various AHL activities

Cultures of the enterobacteria were grown at $37^{\circ} \mathrm{C}$. The rich (LB) and minimal (M9) media were as described previously (Miller, 1972). Aerobic cultures were shaken at 250 r.p.m.; microaerophilic cultures were grown in a stationary anaerobic chamber with $0 \cdot 5 \mathrm{MPa}$ air pressure. Supernatants were collected by removal of bacterial cells from cultures grown to a high cell density, using centrifugation and filtration. AHL activity was measured by adding $10 \%$ of the supernatant to an AHL bioassay strain (Zhu et al., 2003) and $\beta$-galactosidase activity was assayed after $16 \mathrm{~h}$ of growth at $28^{\circ} \mathrm{C}$. Data are presented as Miller units. Basal level $\beta$-galactosidase units (LB or M9 medium control) were $\approx 2$. The results are representative of three experiments and $\mathrm{SD}$ was $\leqslant 15 \%$.

\begin{tabular}{|c|c|c|c|c|}
\hline \multirow[t]{2}{*}{ Strain (no. tested) } & \multicolumn{4}{|c|}{ AHL production (Miller units) } \\
\hline & LB aerobic & LB microaerophilic & M9 aerobic & M9 microaerophilic \\
\hline SRRSH17 & $8 \cdot 3$ & $681 \cdot 4$ & $43 \cdot 6$ & $3 \cdot 5$ \\
\hline SRRSH20 & $7 \cdot 4$ & $231 \cdot 1$ & $29 \cdot 5$ & $25 \cdot 4$ \\
\hline \multicolumn{5}{|l|}{ Klebsiella oxytoca (1) } \\
\hline \multicolumn{5}{|c|}{ Enterobacter aerogenes (2) } \\
\hline SRRSH2 & $6 \cdot 5$ & $69 \cdot 1$ & $3 \cdot 1$ & $4 \cdot 2$ \\
\hline \multicolumn{5}{|c|}{ Enterobacter cloacae (12) } \\
\hline SRRSH59 & $687 \cdot 3$ & $831 \cdot 7$ & $265 \cdot 7$ & $89 \cdot 2$ \\
\hline \multicolumn{5}{|c|}{ Citrobacter freundii (9) } \\
\hline SRRSH27 & $603 \cdot 4$ & $732 \cdot 0$ & $901 \cdot 5$ & $561 \cdot 7$ \\
\hline SRRSH69 & $585 \cdot 3$ & $10 \cdot 1$ & $856 \cdot 4$ & $3 \cdot 5$ \\
\hline \multicolumn{5}{|l|}{ Escherichia coli (12) } \\
\hline \multicolumn{5}{|l|}{ Proteus mirabilis (1) } \\
\hline Morganella morgani & & & & \\
\hline
\end{tabular}

that they had diverse capacities in their AHL-like quorum signal production. Specific strains also produced different amounts of the same AHLs under different environmental conditions. Thus, it seems that quorum sensing may play an important role in the life cycle of commensal enterobacteria, whether they exist asymptomatically in the gut environment or are provoked into causing infection elsewhere. Interestingly, many enterobacteria do not produce any AHLs under laboratory conditions, including the ATCC type strains (except for Serratia; data not shown). This implies that horizontal transfer occurs during intestinal colonization and may have occurred in the clinical isolates possessing AHL synthase genes in this study. This method of quorum gene transfer may be critical to enable certain species to escape their niche as a symbiotic organism and cause infection. In fact, a mobile quorum-sensing system located on a transposon has recently been identified in a strain of $S$. marcescens (Wei et al., 2006).

\section{Acknowledgements}

We thank Dr Chaorong Ge for helping to collect the clinical strains used in this study and Adam Joelsson for reviewing the manuscript. This study is supported by an MOE Major Fund (306009) (to J. Zhu) and

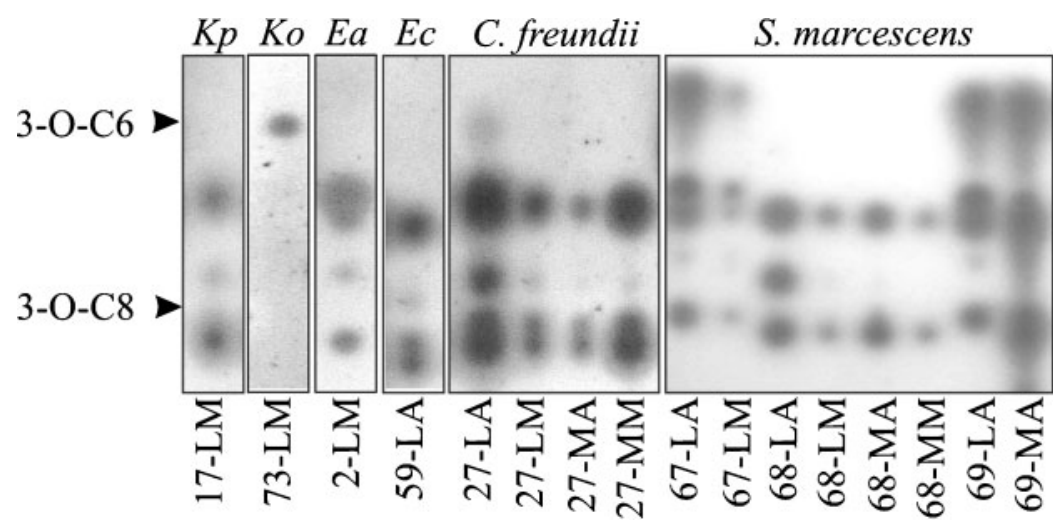

Fig. 1. TLC analysis of $A H L$ production in different enterobacteria. Supernatants from strains grown under LB/aerobic (LA), LB/ microaerophilic (LM), M9/aerobic (MA) or M9/microaerophilic (MM) conditions were extracted with ethyl acetate and applied to C18 reversed-phase TLC plates (Whatman). After separation, the TLC plates were overlaid with agar medium containing $\mathrm{AHL}$ reporter strains and $\mathrm{X}-\mathrm{Gal}$ and incubated overnight at $28^{\circ} \mathrm{C}$ to detect $X-G a l$ hydrolysis. Arrows indicate the positions of synthetic AHLs (3-oxo-C6-HSL and 3-oxo$\mathrm{C} 8-\mathrm{HSL})$ run on the same plate. 


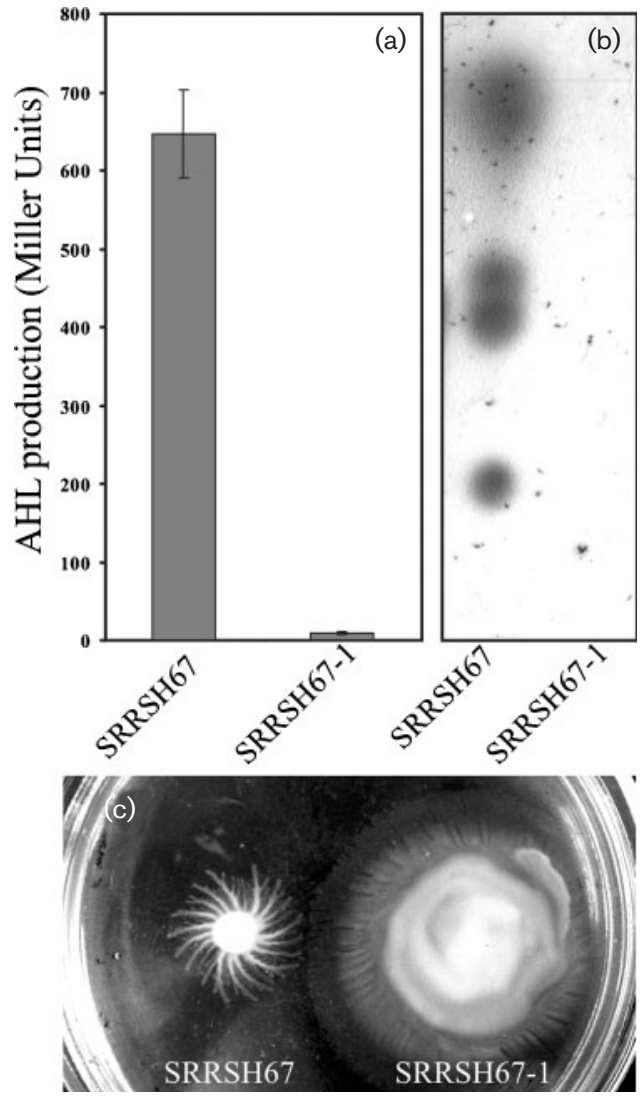

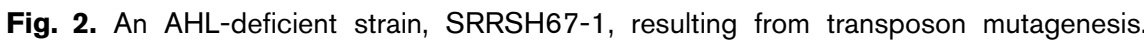
Supernatants of the wild-type and the mutant strain grown in LB medium at $37^{\circ} \mathrm{C}$ were assayed for AHL production (a) and TLC analysis (b). The results are representative of three experiments. Error bars in (a) indicate SD. (c) A quorum-sensing-deficient mutant affects the motility of $S$. marcescens. Both strains were grown on $0.4 \%$ LB agar plates and incubated at $30^{\circ} \mathrm{C}$ overnight.

a Zhejiang DOH grant (2004A052) (to M. Weng).

\author{
Hui Wang, ${ }^{1} \dagger$ Tao Cai, ${ }^{1} \dagger$ \\ Mengwei Weng, ${ }^{2}$ Jing Zhou, ${ }^{1}$ \\ Huijuan Cao, ${ }^{1}$ Zengtao Zhong ${ }^{1}$ \\ and Jun Zhu ${ }^{1,3}$ \\ ${ }^{1}$ Department of Microbiology, MOA Key \\ Laboratory of Microbiological \\ Engineering of the Agricultural \\ Environment, Nanjing Agricultural \\ University, Nanjing, China \\ ${ }^{2}$ Department of Clinic Laboratory, Sir \\ Run Run Shaw Hospital, Zhejiang \\ University College of Medicine, \\ Hangzhou, China
}

\author{
${ }^{3}$ Department of Microbiology, University \\ of Pennsylvania School of Medicine, \\ Philadelphia, USA \\ Correspondence: Jun Zhu \\ (jun_zhu@njau.edu.cn)
}

†These authors contributed equally to this work.

Atkinson, S., Sockett, R. E., Camara, M. \& Williams, P. (2006). Quorum sensing and the lifestyle of Yersinia. Curr Issues Mol Biol 8, 1-10.

Daniels, R., Vanderleyden, J. \& Michiels, J. (2004). Quorum sensing and swarming migration in bacteria. FEMS Microbiol Rev 28, 261-289.

Eberl, L., Winson, M. K., Sternberg, C. \& 7 other authors (1996). Involvement of
$\mathrm{N}$-acyl-L-homoserine lactone autoinducers in controlling the multicellular behaviour of Serratia liquefaciens. Mol Microbiol 20, 127-136. Fuqua, W. C., Winans, S. C. \& Greenberg, E. P. (1994). Quorum sensing in bacteria: the LuxR-LuxI family of cell density-responsive transcriptional regulators. J Bacteriol 176, 269-275.

Horng, Y.-T., Deng, S.-C., Daykin, M. \& 7 other authors (2002). The LuxR family protein SpnR functions as a negative regulator of $\mathrm{N}$-acylhomoserine lactone-dependent quorum sensing in Serratia marcescens. Mol Microbiol 45, 1655-1671.

Judson, N. \& Mekalanos, J. J. (2000).

TnAraOut, a transposon-based approach to identify and characterize essential bacterial genes. Nat Biotechnol 18, 740-745.

Kaper, J. B. \& Sperandio, V. (2005). Bacterial cell-to-cell signaling in the gastrointestinal tract. Infect Immun 73, 3197-3209.

Miller, J. H. (1972). Experiments in Molecular Genetics. Cold Spring Harbor, NY: Cold Spring Harbor Laboratory.

Rubin, E. J., Akerley, B. J., Novik, V. N., Lampe, D. J., Husson, R. N. \& Mekalanos, J. J. (1999). In vivo transposition of mariner-based elements in enteric bacteria and mycobacteria. Proc Natl Acad Sci U S A 96, 1645-1650.

Shaw, P. D., Ping, G., Daly, S. L., Cha, C., Cronan, J. E., Jr, Rinehart, K. L. \& Farrand, S. K. (1997).

Detecting and characterizing $N$-acyl-homoserine lactone signal molecules by thin-layer chromatography. Proc Natl Acad Sci U S A 94, 6036-6041.

Venturi, V. (2006). Regulation of quorum sensing in Pseudomonas. FEMS Microbiol Rev 30, 274-291.

Waters, C. M. \& Bassler, B. L. (2005). Quorum sensing: cell-to-cell communication in bacteria. Annu Rev Cell Dev Biol 21, 319-346.

Wei, J.-R., Tsai, Y.-H., Horng, Y.-T., Soo, P.-C., Hsieh, S.-C., Hsueh, P.-R., Horng, J.-T., Williams, P. \& Lai, H.-C. (2006). A mobile quorum-sensing system in Serratia marcescens. J Bacteriol 188, 1518-1525.

Whitehead, N. A., Barnard, A. M. L., Slater, H., Simpson, N. J. L. \& Salmond, G. P. C. (2001). Quorum-sensing in Gram-negative bacteria. FEMS Microbiol Rev 25, 365-404.

Xavier, K. B. \& Bassler, B. L. (2003). LuxS quorum sensing: more than just a numbers game. Curr Opin Microbiol 6, 191-197.

Zhu, J., Chai, Y., Zhong, Z., Li, S. \& Winans, S. C. (2003). Agrobacterium bioassay strain for ultrasensitive detection of $\mathrm{N}$-acylhomoserine lactone-type quorum-sensing molecules: detection of autoinducers in Mesorhizobium huakuii. Appl Environ Microbiol 69, 6949-6953. 